\title{
ON BOUNDS FOR THE GREATEST CHARACTERISTIC ROOT OF A MATRIX WITH POSITIVE ELEMENTS
}

\author{
GENE W. MEDLIN 1
}

It is known that if $A=\left(a_{k \lambda}\right)$ is a square matrix of order $n$ with positive elements, then the greatest characteristic root $\omega$ of $A$ is positive, simple, and the characteristic vector $X$ of this root may be chosen with all positive components $x_{1}, x_{2}, \cdots, x_{n}[3 ; 4] .^{2}$

Let $x_{r}$ be the greatest and $x_{p}$ be the smallest component of $X$. It is sufficient to assume $x_{r}=1$. A. Ostrowski [5] has just published the following bound for $x_{p}$.

If we set

$$
\begin{aligned}
R_{\lambda} & =\sum_{r=1}^{n} a_{\lambda \nu} \quad \quad(\lambda=1,2, \cdots, n), \\
R & =\max _{\lambda} R_{\lambda} \\
r & =\min _{\lambda} R_{\lambda} \\
K & =\min _{\mu \neq \nu} a_{\mu \nu}
\end{aligned}
$$

then

$$
x_{p}>\frac{K}{R-r+K}=\gamma
$$

for $R \neq r$.

Using the above result we shall improve a theorem of Brauer [2, Theorem 39] for positive matrices. Following Brauer's notation, we let $A=\left(a_{k \lambda}\right)$ be a square matrix of order $n$ with real elements. Assume that all the components of a characteristic vector belonging to the root $\omega$ are positive. Consider the quadratic form

$$
\left(\sum_{\nu=1, v \neq \alpha}^{n} a_{k \nu} y_{\nu}\right)\left(\sum_{\mu=1, \mu \neq \lambda}^{n} a_{\lambda_{\mu}} y_{\mu}\right)
$$

for given $\kappa$ and $\lambda$, and combine the terms which contain the same product $y_{\nu} y_{\mu}$. Denote the sum of the positive coefficients in (1) by $T_{\mathrm{k} \lambda}$ and the sum of the negative coefficients by $t_{\mathrm{k} \lambda}$.

Received by the editors December 16, 1952.

${ }^{1}$ Morehead Scholar, University of North Carolina.

2 Numbers in brackets refer to bibliography at the end of the paper. 
Let

$$
\max \left[\left(T_{k \lambda}-\gamma^{2}\left|t_{k \lambda}\right|\right),\left(\left|t_{k \lambda}\right|-\gamma^{2} T_{k \lambda}\right)\right]=P_{k \lambda} .
$$

TheOREM. Let $A=\left(a_{k \lambda}\right)$ be a square matrix of order $n$ with positive elements and $f_{1}(y), f_{2}(y), \cdots, f_{n}(y)$ polynomials with real coefficients. Let $B=\left(b_{k \lambda}\right)$ be the matrix which has as the vth row the elements of the vth row of $f_{\nu}(A)$. If $P_{\alpha \lambda}^{(B)}$ are the numbers obtained from (1) and (2) for the matrix $B$, then the greatest characteristic root $\omega$ of $A$ satisfies at least one of the inequalities

$$
\left|f_{\alpha}(\omega)-b_{k \alpha}\right|\left|f_{\lambda}(\omega)-b_{\lambda \lambda}\right| \leqq P_{\alpha \lambda}^{(B)} \quad(\kappa, \lambda=1,2, \cdots, n ; \kappa \neq \lambda) .
$$

Proof. The vector $X$ is also a characteristic vector belonging to the characteristic root $f_{v}(\omega)$ of $f_{\nu}(A)$. For proof see Brauer [1, Lemma 2]. We have, therefore,

$$
f_{\nu}(\omega) x_{\nu}=\sum_{\mu=1}^{n} b_{\nu \mu} x_{\mu} \quad(\nu=1,2, \cdots, n)
$$

or

$$
\left(f_{\nu}(\omega)-b_{\nu v}\right) x_{\nu}=\sum_{\mu=1, \mu<\nu}^{n} b_{\nu \mu} x_{\mu} \quad(\nu=1,2, \cdots, n) .
$$

Assume now that $x_{8}$ is such that

$$
x_{r} \geqq x_{s} \geqq x_{\nu} \geqq x_{p} \quad(\nu=1,2, \cdots, n ; \nu \neq r, s, p) .
$$

Multiplying the $r$ th and sth equations of the system (3), we have

$$
\left[f_{r}(\omega)-b_{r r}\right]\left[f_{s}(\omega)-b_{s s}\right] x_{r} x_{s}=\left(\sum_{r=1, v \neq r}^{n} b_{r v} x_{r}\right)\left(\sum_{\mu=1, \mu \neq s}^{n} b_{s \mu} x_{\mu}\right) .
$$

Let $S_{1}$ denote the sum of the positive terms and $S_{2}$ denote the sum of the negative terms in the right-hand side of (4), then

$$
\begin{gathered}
T_{r s} x_{p}^{2} \leqq S_{1} \leqq T_{r s} x_{r} x_{s} \\
\left|t_{r s}\right| x_{p}^{2} \leqq\left|S_{2}\right| \leqq\left|t_{r s}\right| x_{r} x_{s} .
\end{gathered}
$$

Thus

$$
\left|S_{1}+S_{2}\right| \leqq \max \left[\left(T_{r s} x_{r} x_{s}-\left|t_{r s}\right| x_{p}^{2}\right),\left(\left|t_{r s}\right| x_{r} x_{s}-T_{r s} x_{p}^{2}\right)\right] .
$$

Therefore

$$
\begin{aligned}
\left|f_{r}(\omega)-b_{r r}\right| \mid f_{s}(\omega) & -b_{s s} \mid x_{r} x_{s} \\
& \leqq \max \left[\left(T_{r s} x_{r} x_{s}-\left|t_{r s}\right| x_{p}^{2}\right),\left(\left|t_{r s}\right| x_{r} x_{s}-T_{r s} x_{p}^{2}\right)\right] .
\end{aligned}
$$


Since

$$
x_{p}^{2}>\gamma^{2} \geqq x_{r} x_{8} \gamma^{2}
$$

we have

$$
\begin{aligned}
\left|f_{r}(\omega)-b_{r r}\right| \mid f_{s}(\omega)- & b_{s e} \mid x_{r} x_{s} \\
& \leqq x_{r} x_{s} \max \left[\left(T_{r s}-\gamma^{2}\left|t_{r s}\right|\right),\left(\left|t_{r s}\right|-\gamma^{2} T_{r s}\right)\right] .
\end{aligned}
$$

Hence

$$
\left|f_{r}(\omega)-b_{r r}\right|\left|f_{s}(\omega)-b_{s s}\right| \leqq P_{r s}^{(B)} .
$$

It is evident that a similar theorem holds for the columns.

\section{BIBLIOGRAPHY}

1. A. Brauer, Limits for the characteristic roots of a matrix, III, Duke Math. J. vol. 13 (1946) pp. 387-395.

2. - Limits for the characteristic roots of a matrix, V, Duke Math. J. vol. 19 (1952) pp. 553-563.

3. G. Frobenius, Über Matrisen aus positiven Elementen, Preuss. Akad. Wiss. Sitzungsber. (1908) pp. 471-476.

4. - Über Matrizen aus positiven Elementen, Preuss. Akad. Wiss. Sitzungsber. (1909) pp. 514-518.

5. A. Ostrowski, Bounds for the greatest latent root of a positive matrix, J. London ,Math. Soc. vol. 27 (1952) pp. 253-256.

Wake Forest College 